\title{
ANÁLISE DA EFICIÊNCIA DE UTILIZAÇÃO DA LUZ PELA CULTURA DO MILHO SOB DOIS DIFERENTES SISTEMAS DE MANEJO DO SOLO
}

\author{
Claudio A. Teichrieb ${ }^{1}$, Daniel M. dos Santos, Pablo E. S. de Oliveira, Julio C. L. Sena; Hans R. \\ Zimermann, Débora R. Roberti, Osvaldo L.L. de Moraes \\ Laboratório de Micrometeorologia - UFSM - Santa Maria, RS \\ ${ }^{1}$ teichrieb@gmail.com
}

\section{RESUMO}

Neste trabalho, realiza-se a análise da eficiência da utilização da luz pela cultura do milho cultivado com plantio direto e preparo convencional. Relacionou-se a produção primaria bruta obtida pelo método de covariância de vórtices turbulentos, com a radiação fotossinteticamente ativa absorvida pelo dossel das plantas de milho, obtendo-se a eficiência da utilização da luz. A análise mostrou que há uma boa correlação entre a produção primária bruta e a radiação fotossinteticamente ativa absorvida para ambos os sistemas de plantio da cultura do milho. O plantio direto mostrou-se mais eficiente na utilização da luz para a produção primária bruta.

\begin{abstract}
In this work we analyzed the efficiency of light utilization by corn grown under no-tillage and conventional tillage. Related to gross primary production obtained by the method of eddy covariance, with photosynthetically active radiation absorbed by the canopy of corn plants, resulting in the efficient use of light. The analysis showed that there is a good correlation between gross primary production and photosynthetic active radiation absorbed for both tillage systems of corn. The tillage was more efficient in the use of light for gross primary production.
\end{abstract}

\section{INTRODUÇÃO}

O conceito de eficiência na utilização de luz (LUE) é a base subjacente para estimar a troca de carbono em muitos modelos de ecossistema, especialmente os modelos que utilizam o sensoriamento remoto para restringir as estimativas de fotossíntese do dossel (JENKINS, 2007). O entendimento da eficiência na utilização de luz pela vegetação para a produção primária bruta (GPP) é de interesse para aplicação de dados de satélite para monitorar a GPP e a troca líquida de carbono a nível regional para escalas globais (TURNER,2003). O objetivo é avaliar a eficiência de utilização da luz pela cultura do milho em dois diferentes sistemas de preparo do solo. 


\section{METODOLOGIA}

Os dados utilizados neste trabalho foram obtidos de torres micrometeorológicas instaladas em dois diferentes sistemas de manejo do solo para a cultura do milho, o plantio direto (PD) e o preparo convencional (PC). O sítio de estudos está localizado no município de Cruz Alta, RS, nas coordenadas: latitude -28,6036; longitude -53,6736 e altitude média de 432 metros. O sensor utilizado para medir as componentes do vento foi o anemômetro sônico tridimensional - CSAT (Campbell Sci.) e as medidas de $\mathrm{CO}_{2}$ foram obtidas com o analisador de gás infravermelho de caminho aberto - LI7500 (Li-Cor), também, utilizou-se um sensor de radiação fotossinteticamente ativa - modelo PAR LITE (Kipp\&Zonen), com as medidas sendo realizadas a uma taxa de $10 \mathrm{~Hz}$ e armazenadas nesta mesma frequência. Para as medidas do índice de área foliar (LAI - Leaf Area Index), foi utilizado um sensor Li-3000A Portable Area Meter.

Foi utilizada a técnica de eddy covariance para a medida dos fluxos de $\mathrm{CO}_{2}$. Efetuou-se a rotação 3D nas componentes do vento. Aplicou-se a correção de Webb. As médias das variáveis foram calculadas em 30 minutos e em médias diárias. Foram realizadas um total de 8 medidas do LAI com intervalos de 10 a 15 dias cada. As medidas foram feitas em 5 plantas de milho (sempre as mesmas), no PD e PC. Fez-se uma interpolação linear nos dados de LAI, para os cálculos.

Para cada sistema, PD e PC, estimou-se a fração da luz absorvida pelo dossel das plantas de milho, utilizando a lei de Beer-Lambert:

$$
F P A R=0,95(1-\exp (-k \times L A I))
$$

onde FPAR é a fração da luz incidente PAR absorvida pelo dossel (adimensional), $k$ é o coeficiente de extinção da radiação (onde utilizamos $k=0,46$ (DAUGHTRY et al., 1992)) e LAI é o índice de área foliar $\left(\mathrm{m}^{2} \mathrm{~m}^{-2}\right)$. A radiação fotossinteticamente ativa absorvida (APAR) foi calculada como segue:

$$
A P A R=F P A R \times P A R
$$

A eficiência do uso da luz da produção fotossintética (LUE-Light-use efficiency, g C MJ-1) é definida como:

$$
L U E=\frac{G P P}{A P A R}
$$

onde GPP representam a produção primária bruta $\left(\mathrm{g} \mathrm{C} \mathrm{m}^{-2} \mathrm{~d}^{-1}\right)$ e APAR a radiação fotossinteticamente ativa (PAR) absorvida $\left(\mathrm{MJ} \mathrm{m}^{-2} \mathrm{~d}^{-1}\right)$. O conceito LUE foi proposto por Monteith $(1972,1977)$ e é expressa como a quantidade de carbono produzido por unidade de radiação PAR absorvida.

\section{RESULTADOS E DISCUSSÕES}

O cultivo do milho teve início no dia 01 de outubro de 2010 (plantio) e término no dia 05 de março de 2011 (colheita), com duração de 156 dias.

Na figura 1a, podemos observar a evolução do LAI, onde as setas representam os dias em que foram realizadas as medidas. Na figura $1 b$, está representada a evolução da GPP para a cultura do milho. Na figura 1c, observamos o comportamento da radiação PAR absorvida pela cultura. Na figura 1d, mostramos a LUE que pela equação 3 obteve-se a $L U E_{P D}=1,52$ $(0,72) \mathrm{g} \mathrm{C} \mathrm{MJ}^{-1} \mathrm{e} \mathrm{LUE}_{\mathrm{PC}}=1,39(0,74) \mathrm{g} \mathrm{C} \mathrm{MJ}^{-1}$, entre parênteses esta o desvio padrão.

Na figura 2, observamos a correlação linear entre a GPP e a APAR, onde APAR explica $60 \%$ da variação da GPP para o PD e $72 \%$ para o PC. 


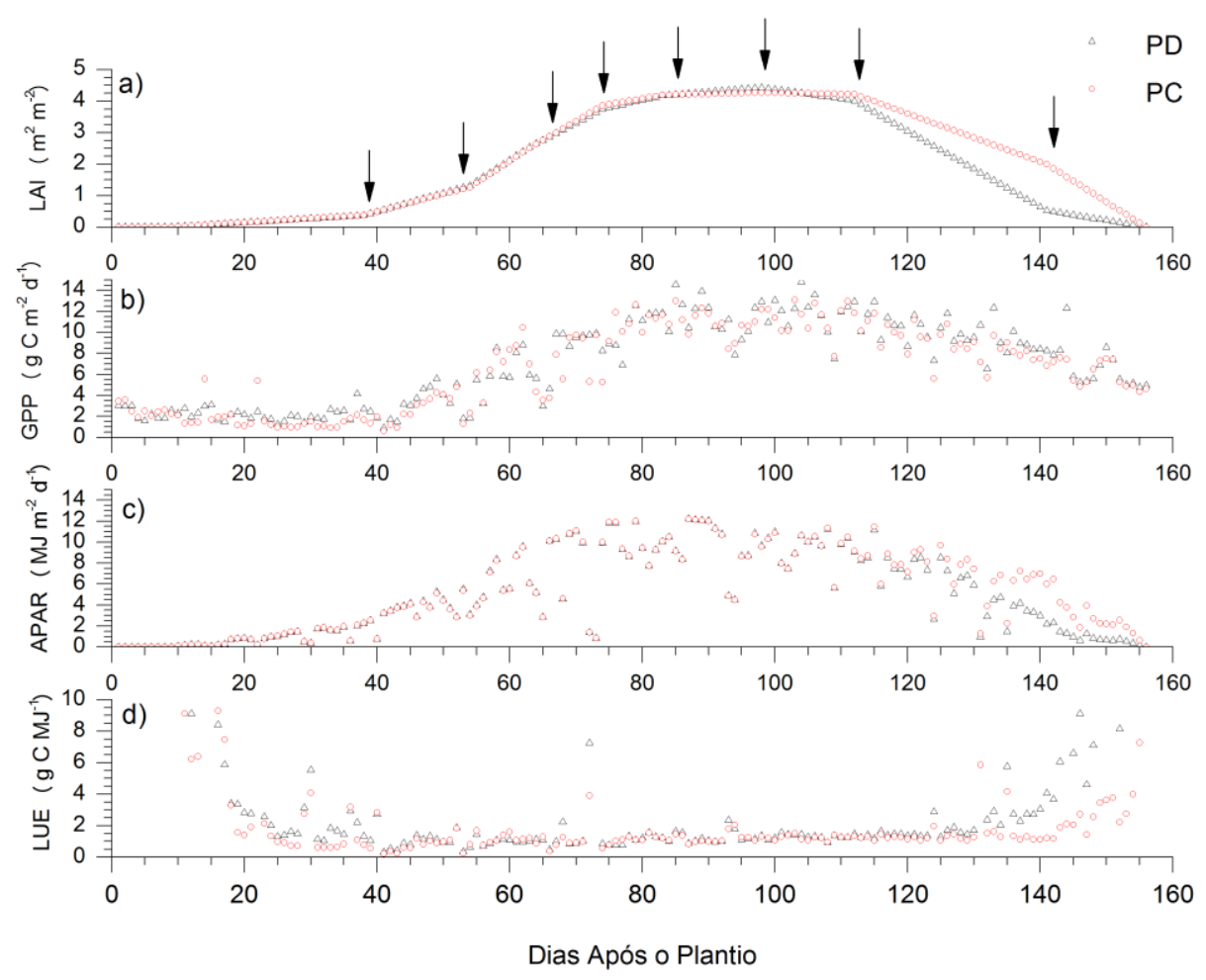

Figura 1. a) Índice de área foliar $\left(\mathrm{m}^{2} \mathrm{~m}^{-2}\right)$; b) Produção primária bruta $\left(\mathrm{g} \mathrm{C} \mathrm{m}^{-2} \mathrm{~d}^{-1}\right)$; c) Radiação fotossinteticamente ativa absorvida $\left(\mathrm{MJ} \mathrm{m}^{-2} \mathrm{~d}^{-1}\right)$ e d) Eficiência de utilização da luz $\left(\mathrm{g} \mathrm{C} \mathrm{MJ}^{-1}\right)$, para o plantio direto (triângulos) e preparo convencional (círculos).
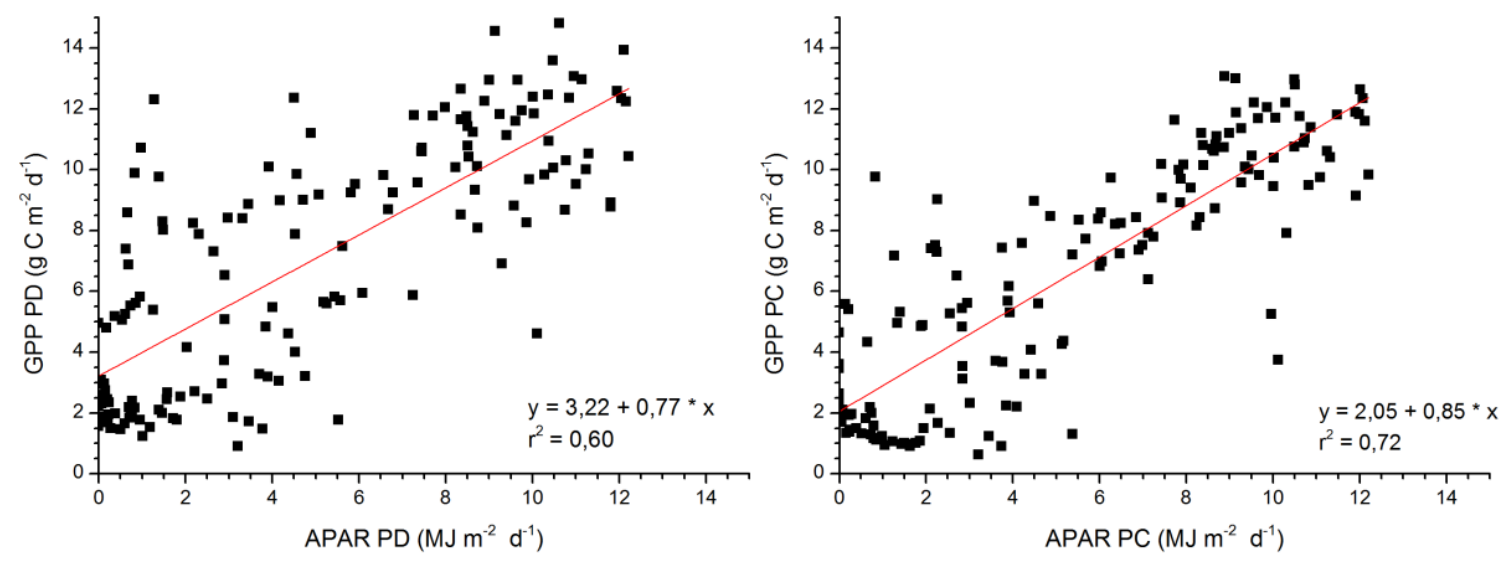

Figura 2. Correlação da produção primária bruta $\left(\mathrm{g} \mathrm{C} \mathrm{m}^{-2} \mathrm{~d}^{-1}\right)$ versus radiação fotossinteticamente ativa absorvida (MJ m $\left.{ }^{-2} \mathrm{~d}^{-1}\right)$, esquerda PD e direita PC. O coeficiente de correlação linear $\left(\mathbf{r}^{2}\right)$, a equação linear contendo a interseção e a inclinação da curva esta indicado.

\section{CONCLUSÕES}

A partir dos resultados, verificou-se que a produção primária bruta e a radiação fotossinteticamente ativa absorvida em uma cultura de milho apresentam uma boa correlação, tendo melhor representação no PC. O PD mostrou ter mais eficiência na utilização da luz pela cultura para a produção primária bruta. 


\section{BIBLIOGRAFIA}

DAUGHTRY, C.S.T. et al. Spectral stimates of absorved radiation and phytomass production in corn and soybean canopies. Remote Sensing of Environmental, 39, 141-152, 1992.

JENKINS, J.P. et al. Refining light-use efficiency calculations for a deciduous forest canopy using simultaneous tower-based carbon flux and radiometric measurements. Agricultural and Forest Meteorology, 143, 64-79, 2007.

MONTEITH, J. L. Solar radiation and productivity in tropical ecosystems. Journal of Applied Ecology. 9, 747-766, 1972

MONTEITH, J. L. Climate and the efficiency of crop production in Britain. Philosophical transactions of the Royal Society of London. Series B, 281, 277-294, 1977.

TURNER, D.P. et al. A cross-biome comparison of daily light use efficiency for gross primary production. Global Change Biology, 9, 383-395, 2003. 